\title{
Magnetic resonance imaging of clinically localized prostatic cancer
}

\section{Ikonen, Saana}

1998

Ikonen , S, Kärkkäinen , P , Kivisaari , L , Salo , J , Taari , K, Vehmas , T , Tervahartiala , P \& Rannikko , S 1998 , ' Magnetic resonance imaging of clinically localized prostatic cancer ' , Journal of Urology , vol. 159 , no. 3 , pp. 915-919 . https://doi.org/10.1016/S0022-5347(01)63770-4

http://hdl.handle.net/10138/297736

https://doi.org/10.1016/S0022-5347(01)63770-4

publishedVersion

Downloaded from Helda, University of Helsinki institutional repository.

This is an electronic reprint of the original article.

This reprint may differ from the original in pagination and typographic detail.

Please cite the original version. 


\title{
MAGNETIC RESONANCE IMAGING OF CLINICALLY LOCALIZED PROSTATIC CANCER
}

\author{
SAANA IKONEN, PÄIVI KÄRKKÄINEN, LEENA KIVISAARI, JAAKKO O. SALO, KIMMO TAARI, \\ TAPIO VEHMAS, PEKKA TERVAHARTIALA AND SAKARI RANNIKKO \\ From the Departments of Radiology and Urology, Helsinki University Central Hospital and Department of Pathology, University of \\ Helsinki, Helsinki, Finland
}

\begin{abstract}
Purpose: We assess the accuracy of endorectal coil magnetic resonance imaging (MRI) for detecting tumor localization, capsular penetration and seminal vesicle invasion in clinically organ confined prostate cancer. We also evaluate intra-observer and interobserver agreement in interpreting MRI studies.

Materials and Methods: MRI studies of 51 consecutive patients a mean of 61 years old with biopsy proved prostate cancer were retrospectively read twice by 2 radiologists in random order. Both radiologists marked tumor localization, capsular penetration and seminal vesicle invasion on standard tumor maps. These findings were compared with the histopathological results of radical prostatectomy specimens.

Results: The overall accuracy of detecting cancer localization was $61 \%$. The detection rate for cancer foci less than $5 \mathrm{~mm}$. was only $5 \%$ but for lesions greater than $10 \mathrm{~mm}$. it was $89 \%$. There was 91 and $80 \%$ accuracy for detecting capsular penetration and seminal vesicle invasion, respectively. Sensitivity and specificity were 60 and 63,13 and 97 , and 59 and $84 \%$ for localization, capsular penetration and seminal vesicle invasion, respectively. Intra-observer and interobserver agreement ranged from fair to good (kappa coefficient 0.240 to 0.647 ).

Conclusions: Endorectal MRI seems to be better than previously reported for detecting seminal vesicle invasion and tumor foci in the anterior half of the prostate. Sensitivity in detecting minor capsular penetration of the tumor was low, which can probably be improved by methodological development. MRI may be useful for locating cancer foci in patients with high prostate specific antigen values but repeatedly negative biopsy findings.
\end{abstract}

KEY WORDS: prostate, magnetic resonance imaging, prostatic neoplasms

The incidence of prostatic cancer is increasing. With the growing use of prostate specific antigen (PSA) testing more early prostate cancer is being detected. ${ }^{1}$ In patients with cancer confined to the gland radical prostatectomy is often recommended as a potential cure. ${ }^{2}$ Therefore, accurate cancer staging at diagnosis is crucial.

The results of staging clinically localized prostate carcinoma with endorectal surface coil magnetic resonance imaging (MRI) have varied considerably. In 1991 Schnall et al reported $82 \%$ accuracy for differentiating stage B from stage $\mathrm{C}$ cancer. ${ }^{3}$ In $1992 \mathrm{Krebs}$ and Silverman described similar findings ${ }^{4}$ but after that time various studies have shown poorer results. Staging accuracy in the series of Quinn ${ }^{5}$ and Tempany ${ }^{6}$ et al was 51 and $54 \%$, respectively, while Perrotti et al achieved $64 \%$ accuracy for identifying periprostatic soft tissue invasion and $77 \%$ accuracy for seminal vesicle invasion. $^{7}$

To stage cancer the lesion must first be located. Recently Jager et al correctly located $67 \%$ of tumors. ${ }^{8}$ Presti et al reported $97 \%$ sensitivity and $58 \%$ specificity for detecting tumor laterality. ${ }^{9}$ Tempany $^{6}$ and Schiebler ${ }^{10}$ et al have shown considerable interobserver variation in interpreting MRI. Hricak et al obtained fair (kappa $=0.38$ ) agreement between consensus and single reader image interpretations. ${ }^{11}$ The main objective of our study was to determine the accuracy of endorectal coil MRI for detecting the localization, capsular penetration and seminal vesicle invasion of prostate tumors by comparing MRI studies to histopathological find-

Accepted for publication August 22, 1997.

Supported by the P. O. Klingendahl Foundation. ings. We also assess intra-observer and interobserver agreement in interpreting MRI studies by having 2 radiologists read them twice in random order.

\section{MATERIALS AND METHODS}

We retrospectively analyzed the MRI studies of 51 consecutive patients 51 to 74 years old (mean age 61) who underwent radical retropubic prostatectomy after imaging. In all patients prostate cancer had been histologically proved by sextant biopsy, and rectal palpation and transrectal ultrasound had shown organ confined disease. Mean serum PSA was $17 \mu \mathrm{g} . / \mathrm{l}$. (range 0 to 100 ). The interval between biopsy and MRI was greater than 3 weeks in all but 2 cases, and the mean interval between MRI and prostatectomy was 29 days (range 1 to 117). Transurethral resection of the prostate had been previously performed in 6 patients and 9 had received hormonal therapy, including finasteride in 2 for benign prostatic hyperplasia and luteinizing hormone-releasing hormone analogue for prostate cancer in 7 .

MRI was performed using a 1.5 Tesla superconducting magnet with a disposable endorectal prostate coil. We obtained T2-weighted fat suppressed turbo spin echo images in the sagittal, axial and coronal directions at 2 acquisitions. The time of repetition was 6,000 msec., echo time was 112 milliseconds and field of view was $150 \times 150 \mathrm{~mm}$. Axial and sagittal images were acquired in $4 \mathrm{~mm}$. thick sections and coronal images were obtained in $3 \mathrm{~mm}$. thick sections with a $1.2 \mathrm{~mm}$. gap. Of the 51 patients 17 were also imaged with an axial 3-dimensional dual echo steady state sequence with repetition time 26.8 milliseconds, echo time $9.0 \mathrm{msec}$, field of 
view $158 \times 180 \mathrm{~mm}$. and slice thickness $2.8 \mathrm{~mm}$. with no gap and 1 acquisition.

MRI studies were retrospectively interpreted twice by 2 of us (L. K. and S. I.) in random order. Radiologist experience in interpreting prostatic endorectal MRI was limited because this technique had only been introduced at our institution the previous year. The interval between the 2 readings was at least 1 month. Radiologists were blinded to the clinical data, although they knew that all patients had biopsy proved, clinically localized prostate cancer.

Based on MRI findings each radiologist marked tumor localization, capsular penetration and seminal vesicle invasion on standard tumor maps on which the prostate was divided into the basis, body and apex (fig. 1). The prostatic body was further divided into 8 segments for more specific tumor localization, and the capsule was divided into the basis, right and left halves, and apex. The seminal vesicles were considered as 1 segment.

Our criterion for cancer in the peripheral zone was a low signal intensity focus. In the central region, consisting of the central and transitional zones, a ground glass-like, homogenous low signal intensity area was the criterion. A localized bulge with an irregular margin or direct tumor extension to periprostatic soft tissues was defined as capsular penetration. The neurovascular bundles were also examined as a possible site of extracapsular extension. The criterion for seminal vesicle invasion was low signal intensity focus in normally bright vesicular tissue. These criteria were derived from the literature ${ }^{5,11,12}$ and our experience.

Radical retropubic prostatectomy was performed in all patients and modified pelvic lymphadenectomy was also done to exclude pelvic lymph node metastases. After surgical removal the intact prostate and seminal vesicles were coated over the whole external surface with silver and fixed in formalin for 2 to 4 days. After fixation the right prostatic lobe was marked with an incision. The seminal vesicles, and apical and basal urethral margins were removed for histological evaluation. The remaining whole prostate was step sectioned at $5 \mathrm{~mm}$. intervals. All sections were designated to permit localization of each section within the prostate. After paraffin embedding all slides were stained according to the Herovicivan Gieson method. Sections were examined by a single pathologist (P. K.) and cancer areas were outlined with a pen.

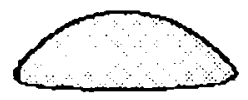

basis

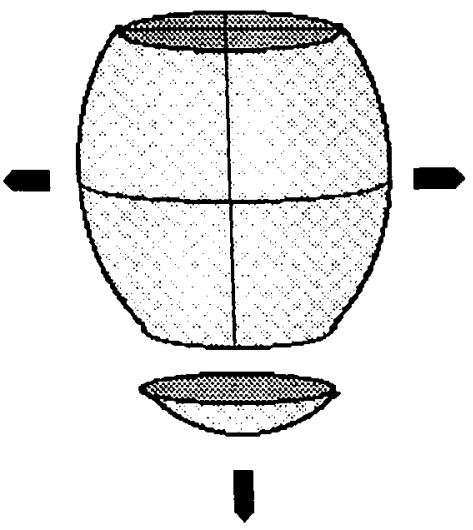

body of the prostate ( 8 segments)

Fig. 1. Division of prostate gland and capsule. Arrows indicate capsular penetration.
These microscopic findings were then marked on standard tumor maps. Tumor maps drawn by the radiologists and the pathologist were compared segment by segment in regard to cancer localization, capsular penetration and seminal vesicle invasion.

Contingency $(2 \times 2)$ tables showed the association between MRI and histopathological findings. We calculated binomial 95\% confidence intervals (CI) for accuracy, sensitivity and specificity for capsular penetration and seminal vesicle invasion because the number of positive cases was low. To evaluate intra-observer and interobserver agreement we used kappa statistics, and we interpreted kappa values according to Altman. ${ }^{13}$ We performed the chi-square test with the Yates correction for continuity to compare accuracy, sensitivity and specificity of MRI diagnoses in the hormone treated and nontreated, and transurethral prostatic resection and nonresection groups. Due to multiple comparisons (6) the limit for significance was considered at $p=0.01$ according to the Bonferroni method. ${ }^{14}$

\section{RESULTS}

Overall accuracy for detecting tumor localization was $61 \%$, sensitivity was $60 \%$ and specificity was $63 \%$ (table 1). There were considerable differences for detecting tumor foci in various parts of the prostate (table 2). Localizing cancer foci was more difficult in the basis and apex than in the body of the prostate with 58,41 and $64 \%$ accuracy, respectively. Detecting tumors in the anterior half of the prostate was less accurate than in the posterior half with 61 and $67 \%$ accuracy, respectively.

To estimate how well MRI detected small cancer foci we counted the lesions less than $5 \mathrm{~mm}$. on the tumor maps of all patients. The pathologist had marked 140 lesions and the radiologists had marked 7 . We also counted lesions greater than $10 \mathrm{~mm}$., of which the pathologist had found 62 and the radiologists had found 55 .

In 9 patients who had received hormonal treatment before MRI there was $56 \%$ accuracy for detecting cancer localization compared to $62 \%$ in those who did not undergo hormonal treatment (not statistically significant). There were similar findings in the groups that did and did not undergo transurethral prostatic resection (table 3 ).

Pathological evaluation revealed extraprostatic tumor extension in 12 of the 51 patients ( $24 \%, 14$ of 204 possible sites). The overall accuracy for discovering penetration was $91 \%$ (95\% CI 87 to 95 ). Sensitivity was poor at only $13 \%$ (95\% CI 2 to 43 ), whereas specificity reached 97\% (94 to 99). There were a mean of 2 positive, 184 negative, 5 false-positive and 12 false-negative cases (table 4).

Pathological analysis also showed tumor invasion into the seminal vesicles in 8 patients $(16 \%)$. There were a mean of 5 positive, 36 negative, 7 false-positive and 3 false-negative cases. For detecting invasion accuracy was $80 \%$ (95\% CI 67 to 90 ), sensitivity was $59 \%$ (24 to 91 ) and specificity was $84 \%$ (69 to 93 ) (table 5). Clinically 17 cases $(33 \%)$ were under staged. When we compared the groups with stages T2 and T3 disease as interpreted by the radiologists and pathologist, we found $69 \%$ staging accuracy (table 6). Intra-observer and

TABLE 1. Tumor localization

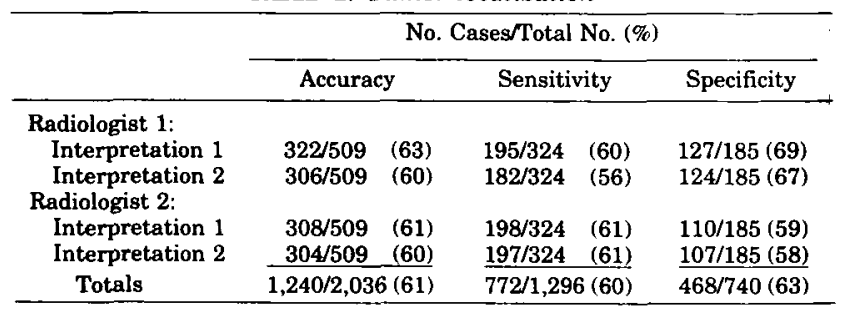


TABLE 2. Tumor localization in different parts of the prostate

\begin{tabular}{|c|c|c|c|}
\hline \multirow{2}{*}{ Prostate Part } & \multicolumn{3}{|c|}{ No. Cases/Total No. (\%) } \\
\hline & Accuracy & Sensitivity & Specificity \\
\hline Body & $1,040 / 1,632(64)$ & $705 / 1,040(68)$ & $335 / 592(57)$ \\
\hline Basis & $118 / 204 \quad(58)$ & $20 / 92$ & $98 / 112(88)$ \\
\hline Apex & $82 / 200^{*}(41)$ & $47 / 164 \quad(29)$ & $35 / 36 \quad(97)$ \\
\hline Anterior half & $495 / 816 \quad(61)$ & $244 / 444 \quad(55)$ & $251 / 372(67)$ \\
\hline Posterior half & $545 / 816 \quad(67)$ & $461 / 596 \quad(77)$ & $84 / 220(38)$ \\
\hline
\end{tabular}

* One apex section was missing.

TABLE 3. The effect of hormonal therapy and transurethral prostatic resection on localization of prostate cancer

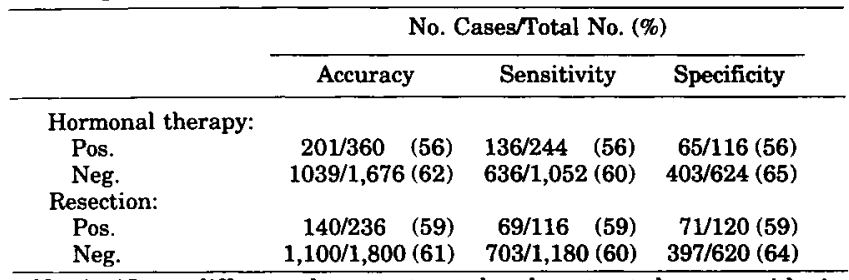

No significant difference between treated and nontreated groups with significance considered at $\mathrm{p}=0.01$.

TABLE 4. Capsular penetration

\begin{tabular}{clcc}
\hline & \multicolumn{3}{c}{ No. Cases/Total No. (\%) } \\
\cline { 2 - 4 } & Accuracy* $^{*}$ & Sensitivity & Specificity \\
\hline Radiologist 1: & & & \\
Interpretation 1 & $182 / 203(90)$ & $1 / 14(7)$ & $181 / 189(96)$ \\
Interpretation 2 & $187 / 203(92)$ & $2 / 14(14)$ & $185 / 189(98)$ \\
Radiologist 2: & & & \\
Interpretation 1 & $189 / 203(93)$ & $2 / 14(14)$ & $187 / 189(99)$ \\
Interpretation 2 & $\underline{184 / 203(91)}$ & $\underline{2 / 14(14)}$ & $\underline{182 / 189(96)}$ \\
$\quad$ Totals & $742 / 812(91)$ & $7 / 56(13)$ & $735 / 756(97)$ \\
\hline
\end{tabular}

* One apex section was missing.

TABLE 5. Seminal vesicle invasion

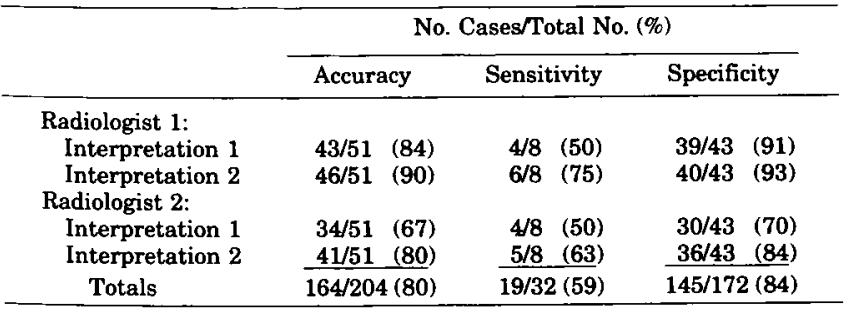

interobserver consistencies were calculated for detecting cancer localization, capsular penetration and seminal vesicle invasion, and agreement rates ranged from fair to good (table 7).

\section{DISCUSSION}

In all of our patients biopsy proved cancer was confined to the prostate based on palpation and transrectal ultrasound. Salo et al showed that transrectal ultrasound is highly accurate for revealing local cancer extension beyond the prostatic capsule. ${ }^{15}$ Thus, our patient population was highly selected and our results concern only clinically localized disease. We excluded patients with clear extraprostatic growth because they did not undergo radical prostatectomy, and so pathological verification was not available. This patient selection biased the sensitivity and specificity of capsular penetration and seminal vesicle invasion. The knowledge of clinically localized disease may also have influenced image interpretation.

Although we were aware of the possibility that changes
TABlE 6. Stage T2 versus T3 disease

\begin{tabular}{ccccc}
\hline & \multicolumn{4}{c}{ No. Cases/Total No. (\%) } \\
\cline { 2 - 5 } & \multicolumn{1}{c}{ Accuracy } & Sensitivity & \multicolumn{1}{c}{ Specificity } \\
\hline Radiologist 1: & & & & \\
Interpretation 1 & $33 / 51$ & $(65)$ & $7 / 17(41)$ & $26 / 34(76)$ \\
Interpretation 2 & $39 / 51(76)$ & $9 / 17(53)$ & $30 / 34(88)$ \\
Radiologist 2: & & & & \\
Interpretation 1 & $33 / 51(65)$ & $9 / 17(53)$ & $24 / 34(71)$ \\
Interpretation 2 & $36 / 51(71)$ & $\underline{10 / 17(59)}$ & $\underline{26 / 34(76)}$ \\
Totals & $141 / 204(69)$ & $35 / 68(51)$ & $106 / 136(78)$ \\
\hline
\end{tabular}

TABLE 7. Kappa values of intra-observer and interobserver agreement rates

\begin{tabular}{lccc}
\hline & $\begin{array}{c}\mathrm{Ca} \\
\text { Localization }\end{array}$ & $\begin{array}{c}\text { Capsular } \\
\text { Penetration }\end{array}$ & $\begin{array}{c}\text { Seminal Vesicle } \\
\text { Invasion }\end{array}$ \\
\hline Intra-observer: & & & \\
Radiologist 1 & 0.576 & 0.240 & 0.647 \\
Radiologist 2 & 0.462 & 0.446 & 0.381 \\
Interobserver radiologist 1 vs. 2 & 0.421 & 0.240 & 0.463 \\
\hline Kappa values were interpreted as poor-0.20 or less, fair-0.21 to 0.40, \\
moderate- 0.41 to 0.60 , good- 0.61 to 0.80 and very good- 0.81 to 1.00 .
\end{tabular}

after biopsy interfere with image interpretation, there was no T1 sequence in our imaging protocol, because the average time between biopsy and MRI in our study was greater than 3 weeks. Recently White et al reported that, when imaging was deferred for 21 days after biopsy, staging accuracy significantly improved. ${ }^{16}$ In addition, imaging time was already prolonged due to axial, sagittal and coronal imaging directions and, thus, an even longer imaging time would likely have resulted in more motion artifacts. However, by using a $\mathrm{T} 1$ sequence we may have been able to decrease false-positive findings caused by post-biopsy hematoma.

Another problem with imaging was that fat suppression of T2 images may have limited our detection of tumor outgrowth. However, we had to use this method because of the bright signal from fat in turbo spin echo imaging. To resolve this problem we added a 3-dimensional dual echo steady state sequence to our protocol. We did not separately evaluate the 17 patients who underwent MRI using both sequences because pathological evaluation revealed capsular penetration in only 3 . We also believe that using even thinner imaging slices ( 1.5 to $2.0 \mathrm{~mm}$.) would improve the detection of capsular penetration and localization of small cancer foci.

Previous studies have focused on evaluating the staging accuracy of MRI in prostatic cancer. In addition to evaluating staging accuracy, we assessed the ability of MRI to localize cancer foci. Our results suggest that accuracy in detecting lesions depends on the location in the prostate.

Outwater et al identified none of the 29 central gland tumors in their study. ${ }^{17}$ Carter et al reported $15 \%$ sensitivity for tumors located anteriorly versus $85 \%$ for those situated posteriorly. ${ }^{18}$ Our corresponding values were 55 and $77 \%$, respectively. The improved identification of anterior tumors in our study was probably due to the use of a separate criterion (ground glass-like, homogenous low signal intensity area) for cancer in the central gland. However, stromal hyperplasia can mimic cancer (figs. 2 and 3), sometimes resulting in false-positive findings.

To our knowledge no previous studies have described difficulty in localizing tumors in the prostatic apex or basis. Our results in the apex region were poor, although we also used sagittal and coronal imaging directions, in addition to axial images, to decrease partial volume effects. More attention should be paid to this area, especially since a tumor involving the apex can readily invade the extraprostatic tissues. ${ }^{5,19}$ Detecting cancer foci in the basis was more difficult than detecting those in the prostatic body. This finding may be important, because cancer usually invades the seminal vesicles by direct tumor spread from the mid base region near 

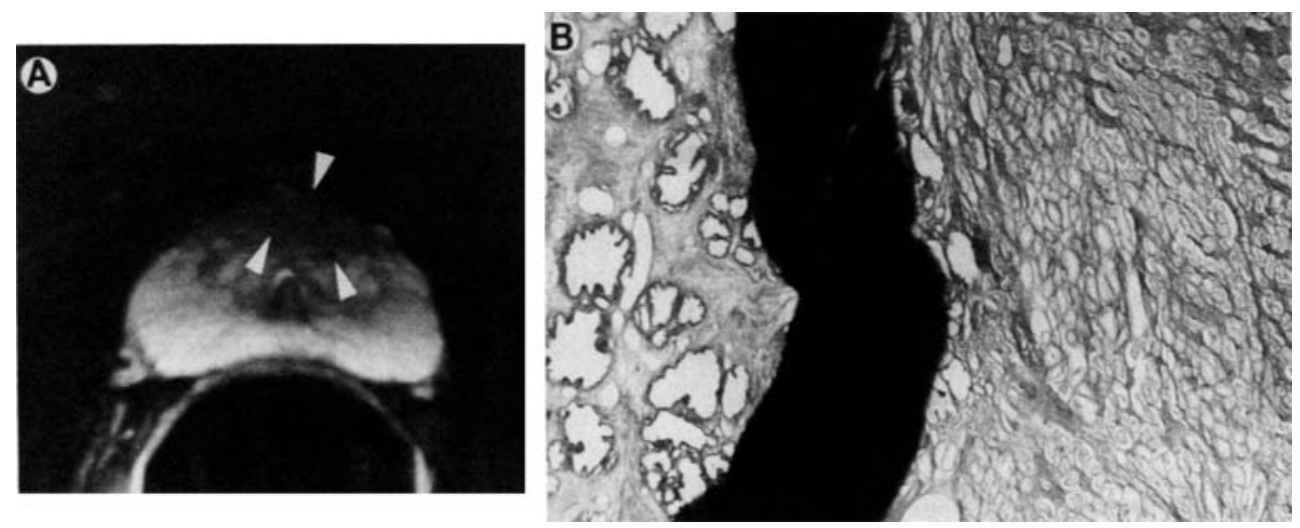

Fig. 2. A, axial T2-weighted image shows carcinoma in anterior part of prostate (arrowheads). $B$, same tumor tissue seen on right side, normal glands on left side. Reduced from $\times 20$.
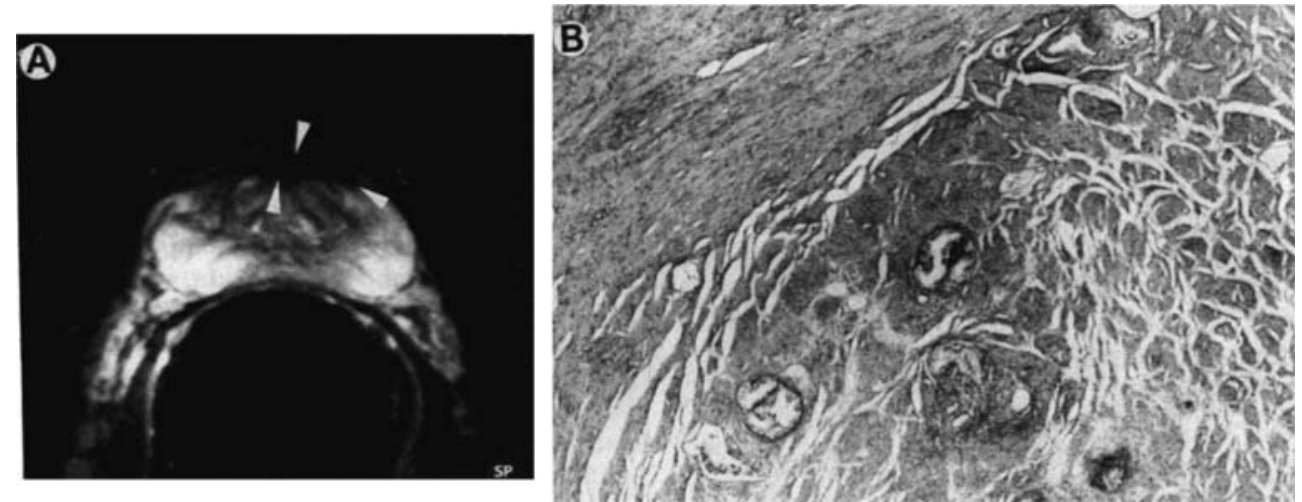

FIG. 3. A, axial T2-weighted image shows stromal hyperplasia in anterior part of prostate (arrowheads). $B$, same stromal hyperplasia (upper left side). Reduced from $\times 40$.

the ejaculatory ducts, ${ }^{20}$ and seminal vesicle invasion has a significantly adverse effect on prognosis. ${ }^{21}$

Prostate cancer often consists of many small lesions. The modest value of sensitivity $(60 \%)$ in our study may have been caused by difficulty in detecting diffuse multifocal types of prostatic cancer. On MRI we noted only $5 \%$ of tumor foci less than $5 \mathrm{~mm}$. in contrast to the histopathological findings. On the other hand, such small tumors are not likely to extend to the extracapsular tissues. According to Stamey et al, $80 \%$ of tumors less than $0.5 \mathrm{ml}$. are unlikely to be significant during the life of the patient. ${ }^{22}$ We detected $89 \%$ of the tumor foci greater than $10 \mathrm{~mm}$. Other prostatic diseases, such as stromal hyperplasia or inflammatory foci, may have deleteriously affected specificity $(63 \%)$ because they may resemble cancer.

Our results support those of Schiebler ${ }^{22}$ and Chen $^{23}$ et al that hormonal therapy may alter MRI findings. However, there was no statistically significant difference in cancer detection accuracy between the treated and nontreated groups. Quinn et al reported that previous transurethral prostatic resection may make tumor delineation difficult but we observed no corresponding trend. ${ }^{5}$

Compared to previous studies ${ }^{3-7}$ our overall $69 \%$ staging accuracy seems moderate. Epstein et al showed that established capsular penetration in prostatectomy specimens indicates a higher risk of progression than in those with focal capsular penetration (few neoplastic glands outside the prostate). ${ }^{24}$ We made no distinction between microscopic and macroscopic capsular penetration, because at many sites the surgical margins surrounding the specimen were thin and, thus, no stages of macroscopic penetration were determined.
However, extracapsular extension in this material was mainly microscopic. This situation as well as the small number of extracapsular penetrations were caused by the fact that our patients had clinically localized disease, which affected sensitivity and specificity. The small number of pathologically verified positive cases caused some uncertainty in the results. In our series there were poor (13\%) sensitivity for capsular penetration, $97 \%$ specificity and $91 \%$ overall accuracy. Perrotti et al reported $22 \%$ sensitivity, $84 \%$ specificity and $64 \%$ accuracy with no distinction made between microscopic and macroscopic penetration. ${ }^{7}$ In the series of Jager et al sensitivity was $14 \%$ when penetration was less than 1 mm., $67 \%$ when it was 1 to $3 \mathrm{~mm}$. and $100 \%$ when it was greater than $3 \mathrm{~mm}^{8}$

For detecting seminal vesicle invasion Jager et $\mathrm{al}^{8}$ reported $36 \%$ sensitivity but only $9 \%$ specificity. The study of Perrotti et al also had low sensitivity $(23 \%)$ but better specificity $(93 \%),{ }^{7}$ while Tempany et al reported values of 21 and $85 \%$, respectively. ${ }^{6}$ Our corresponding values were 59 and $84 \%$ with clearly better sensitivity than in previous studies. Unfortunately 7 false-positive cases were also found. All of our patients had undergone transrectal ultrasound before MRI and were supposed to have localized disease based on ultrasound findings. However, MRI revealed seminal vesicle invasions, and so MRI seems to be more sensitive than transrectal ultrasound in this respect.

Our results show no difference from those of previous studies in regard to considerable interobserver variation in interpreting MRI. ${ }^{6,10,11}$ For tumor localization intra-observer and interobserver agreements were moderately consistent. Agreement between readers was weakest in detecting capsu- 
lar penetration and best in detecting seminal vesicle invasion. Consensus training in the interpretation of MRI may improve results. Generally intra-observer agreement was somewhat better than interobserver agreement. Partial improvement on the second reading was probably caused by our pilot series, which was analyzed during the study period.

When we started this study in 1995 contrast enhancement was considered to be of no particular use in MRI for prostatic cancer. Jager et al have now reported better results using the gadolinium enhanced dynamic subtracted technique compared with T2-weighted fast spin echo images. ${ }^{25}$ Sensitivity, specificity and accuracy for detecting tumor involvement on enhanced images were $73.5,81.0$ and $77.5 \%$ compared with $57.5,80.5$ and $72.0 \%$ on fast spin echo images, respectively. The depiction of capsular penetration and tumor staging were also better when enhanced images were included. However, there was no statistically significant difference between the 2 sequences. There are also new studies of prostatic MRI using pelvic phased array coils. Husband et al reported that pelvic phased array coil imaging visualizes the anterior gland and neurovascular bundles better than endorectal coil imaging. ${ }^{26}$ In their study there was no difference in tumor staging. Earlier Hricak et al noted better results using integrated endorectal pelvic phased array coils than phased array coils only. ${ }^{11}$ The further development of MRI techniques for imaging the prostate will probably improve results in the future.

\section{CONCLUSIONS}

MRI seems to be more sensitive for detecting seminal vesicle invasion than transrectal ultrasound. Sensitivity for detecting minor capsular penetration was low but it may be improved by dynamic contrast enhancement and new coil technology. Identifying cancer foci in the anterior half of the prostate was more reliable than previously reported. The detection rate of tumors greater than $10 \mathrm{~mm}$. was good, and so MRI may be useful for locating cancer foci in patients with high PSA values but repeatedly negative biopsy findings.

\section{REFERENCES}

1. Mettlin, C. J., Murphy, G. P., McGinnis, L. S. and Menck, H. R.: The national cancer data base report on prostate cancer. Cancer, 76: 1104, 1995.

2. Middleton, R. G., Thompson, I. M., Austenfeld, M. S., Cooner, W. H., Correa, R. J., Gibbons, R. P., Miller, H. C., Oesterling, J. E., Resnick, M. I., Smalley, S. R. and Wasson, J. H.: Prostate cancer clinical guidelines panel summary report on the management of clinically localized prostate cancer. J. Urol., 154: $2144,1995$.

3. Schnall, M. D., Imai, Y., Tomaszewski, J., Pollack, H. M., Lenkinski, R. E. and Kressel, H. Y.: Prostate cancer: local staging with endorectal surface coil MR imaging. Radiology, 178: $797,1991$.

4. Krebs, T. L. and Silverman, J. M.: Clinical utility of endorectal surface coil MR imaging of the prostate gland. Radiology, 185P: 275, abstract, 1992 .

5. Quinn, S. F., Franzini, D. A., Demlow, T. A., Rosencrantz, D. R., Kim, J., Hanna, R. M. and Szumowski, J.: MR imaging of prostate cancer with an endorectal surface coil technique: correlation with whole-mount specimens. Radiology, 190: 323, 1994.

6. Tempany, C. M., Xiao, Z., Zerhouni, E. A., Rifkin, M. D., Quint, L. E., Piccoli, C. W., Ellis, J. H. and McNeil, B. J.: Staging of prostate cancer: results of Radiology Diagnostic Oncology Group project comparison of three MR imaging techniques. Radiology, 192: 47, 1994.

7. Perrotti, M., Kaufman, R. P., Jennings, T. A., Thaler, H. T., Soloway, S. M., Rifkin, M. D. and Fisher, H. A. G.: Endo-rectal coil magnetic resonance imaging in clinically localized prostate cancer: is it accurate? J. Urol., 156: 106, 1996.

8. Jager, G. J., Ruijter, E. T.: G., van de Kaa, C. A., de la Rosette, J. J. M. C. H., Oosterhof, G. O. N., Thornbury, J. R. and
Barentsz, J. O.: Local staging of prostate cancer with endorectal MR imaging: correlation with histopathology. AJR, 166: $845,1996$.

9. Presti, J. C., Jr., Hricak, H., Narayan, P. A., Shinohara, K, White, S. and Carrol, P. R.: Local staging of prostatic carcinoma: comparison of transrectal sonography and endorectal MR imaging. AJR, 166: 103, 1996.

10. Schiebler, M. L., Yankaskas, B. C., Tempany, C., Spritzer, C. E., Rifkin, M. D., Pollack, H. M., Holtz, P. and Zerhouni, E. A.: MR imaging in adenocarcinoma of the prostate: interobserver variation and efficacy for determining stage C disease. AJR, 158: $559,1992$.

11. Hricak, H., White, S., Vigneron, D., Kurhanewicz, J., Kosco, A., Levin, D., Weiss, J., Narayan, P. and Carroll, P. R.: Carcinoma of the prostate gland: MR imaging with pelvic phased-array coils versus integrated endorectal-pelvic phased-array coils. Radiology, 193: 703, 1994

12. Schiebler, M. L., Schnall, M. D., Pollack, H. M., Lenkinski, R. E., Tomaszewski, J. E., Wein, A. J., Whittington, R., Rauschning, W. and Kressel, H. Y.: Current role of MR imaging in the staging of adenocarcinoma of the prostate. Radiology, 189: 339, 1993.

13. Altman, D. G.: Practical Statistics to Medical Research. London: Chapman \& Hall, p. 404, 1991.

14. Glantz, S. A.: Primer of Biostatistics. Singapore: McGraw-Hill, pp. 139-140, 1997.

15. Salo, J. O., Kivisaari, L., Rannikko, S. and Lehtonen, T.: Computerized tomography and transrectal ultrasound in the assessment of local extension of prostatic cancer before radical retropubic prostatectomy. J. Urol., 137: 435, 1987.

16. White, S., Hricak, H., Forstner, R., Kurhanewicz, J., Vigneron, D. B., Zaloudek, C. J., Weiss, J. M., Narayan, P. and Carroll, P. R.: Prostate cancer: effect of postbiopsy hemorrhage on interpretation of MR images. Radiology, 195: 385, 1995.

17. Outwater, E. K., Petersen, R. O., Siegelman, E. S., Gomella, L. G., Chernesky, C. E. and Mitchell, D. G.: Prostate carcinoma: assessment of diagnostic criteria for capsular penetration on endorectal coil MR images. Radiology, 193: 333, 1994.

18. Carter, H. B., Brem, R. F., Tempany, C. M., Yang, A., Epstein, J. I., Walsh, P. C. and Zerhouni, E. A.: Nonpalpable prostate cancer: detection with MR imaging. Radiology, 178: 523, 1991.

19. Villers, A. A., McNeal, J. E., Redwine, E. A., Freiha, F. S. and Stamey, T. A.: The role of perineural space invasion in the local spread of prostatic adenocarcinoma. J. Urol., 142: 763, 1989.

20. Villers, A. A., McNeal, J. E., Redwine, E. A., Freiha, F. S. and Stamey, T. A.: Pathogenesis and biological significance of seminal vesicle invasion in prostatic adenocarcinoma. J. Urol., 143: 1183, 1990.

21. Epstein, J. I., Pizov, G. and Walsh, P. C.: Correlation of pathologic findings with progression after radical retropubic prostatectomy. Cancer, 71: 3582, 1993.

22. Stamey, T. A., Freiha, F. S., McNeal, J. E., Redwine, E. A. Whittemore, A. S. and Schmid, H.-P.: Localized prostate cancer. Relationship of tumor volume to clinical significance for treatment of prostate cancer. Cancer, 71: 933, 1993.

23. Chen, M., Hricak, H., Kalbhen, C. L., Kurhanewicz, J,. Vigneron, D. B., Weiss, J. M. and Carroll, P. R.: Hormonal ablation of prostatic cancer: effects on prostate morphology, tumor detection, and staging by endorectal coil MR imaging. AJR, 166: $1157,1996$.

24. Epstein, J. I., Carmichael, M. J., Pizov, G. and Walsh, P. C.: Influence of capsular penetration on progression following radical prostatectomy: a study of 196 cases with long-term followup. J. Urol., 150: 135, 1993.

25. Jager, G. J., Ruijter, E. T. G., vd Kaa, C.: A., de la Rosette, J. J. M. C. H., Oosterhof, G. O. N., Thornbury, J. R., Ruijs, S. H. J. and Barentsz, J. O.: Dynamic TurboFLASH subtraction technique for contrast-enhanced MR imaging of the prostate: correlation with histopathologic results. Radiology, 203: 645, 1997.

26. Husband, J. E., Padhani, A. R., MacVicar, D.and Revell, P.: MR of prostate cancer. Comparison of image quality using pelvic phased array and endorectal coils. Adv. MRI, No. 095, Second International Magnetom Vision Conference, abstract, Rotterdam, 1997. 